\title{
Antipsychotic agent pimozide promotes reversible proliferative suppression by inducing cellular quiescence in liver cancer
}

\author{
JIA-JIE CHEN $^{1 *}$, LI-NA ZHANG $^{1 *}$, NAN CAI $^{2}$, ZHEN ZHANG $^{1}$ and KUNMEI JI ${ }^{1}$ \\ ${ }^{1}$ Department of Biochemistry and Molecular Biology, School of Medicine, ${ }^{2}$ College of Life Science and Oceanography, \\ Shenzhen University, Shenzhen, Guangdong 518060, P.R. China
}

Received February 21, 2019; Accepted July 8, 2019

DOI: $10.3892 /$ or.2019.7229

\begin{abstract}
The antipsychotic drug pimozide has been found to exhibit anticancer effects. Previously, it was demonstrated that pimozide inhibits hepatocellular carcinoma (HCC) cell growth, but its pharmacodynamic characteristics remain unclear. The aim of the present study was to investigate the reversibility and mechanism of the ability of pimozide to inhibit cell proliferation in liver cancer. Cell viability was determined by Cell Counting Kit- 8 and colony formation assay. The cell cycle distribution was analyzed by flow cytometry with Ki-67 and PI staining. ROS production of HCC cells was detected with DCFH-DA and inhibited with NAC treatment. Western blot assay was performed to detect the expression of related signaling molecules in HCC cells. Our results showed that pimozide promoted $\mathrm{G} 0 / \mathrm{G} 1$ phase arrest in $\mathrm{HCC}$ cell lines without significant cell death. Its anti-proliferative effects on HCC cells were reversible, consistent with involvement of cell quiescence and reactive oxygen species (ROS) production. Pimozide enhanced inhibition of HCC cell proliferation by sorafenib. In conclusion, elucidation of pimozide's reversible proliferation inhibition in liver cancer and additive activity with a well-established anticancer drug warrants further exploration of the potential of pimozide as an adjuvant anticancer therapy.
\end{abstract}

\section{Introduction}

Hepatocellular carcinoma (HCC) is one of the most prevalent and lethal malignancies in the world (1), with a 5-year

Correspondence to: Dr Jia-Jie Chen or Professor Kunmei Ji, Department of Biochemistry and Molecular Biology, School of Medicine, Shenzhen University, 1066 Xueyuan Road, Nanshan District, Shenzhen, Guangdong 518060, P.R. China

E-mail: chenjj@szu.edu.cn

E-mail: jkm@szu.edu.cn

*Contributed equally

Key words: pimozide, hepatocellular carcinoma, quiescence, reversible proliferation inhibition, drug repurposing survival rate of only $30-40 \%$ (2). HCC has a poor prognosis due to chemotherapy resistance and a high recurrence rate (3). Given the unsatisfactory state of current liver cancer treatment effectiveness, there is a great need for the development of new strategies for targeting HCC (4).

Drug repurposing is an important approach in cancer drug discovery and development $(5,6)$. Notably, we previously found that pimozide, an FDA-approved compound used to treat chronic psychosis, exhibits anticancer effects on various types of carcinomas, including HCC (7), prostate cancer (8) and osteosarcoma (9). Pimozide has been reported to inhibit cell proliferation, colony formation, and sphere formation of these three cancer types (7-9). Pimozide also reduced migration of prostate and liver cancer cells (7). Additionally, pimozide increased the sensitivity of breast cancer cells to $\gamma$-irradiation treatment (10) and enhanced the anticancer effect of L-methyl-tryptophan (an indoleamine 2, 3-dioxygenase inhibitor) on melanoma cells (11). Furthermore, previously, we found that pimozide inhibited cancer cell growth through suppression of signal transducer and activator of transcription 3 (STAT3) activation (7-9) or Wnt/3-catenin signaling $(12,13)$. In addition, pimozide has been shown to inhibit maintenance and tumorigenicity of HCC stem-like cells (14) and to induce reactive oxygen species (ROS) generation, which can impede cell proliferation (9).

In light of the aforementioned findings, the potential clinical anticancer efficacy of pimozide has piqued our interest. The toxicology profile of pimozide in human patients is well established owing to its long-term use in psychiatry. In addition, molecular targets involved in the suppression of cancer cell growth by pimozide have been identified. However, various factors affecting the drug's pharmacodynamic effects remain to be clarified, including whether the suppression of HCC cell proliferation by pimozide is reversible. Irreversible mechanisms include cell death, cell senescence and terminal differentiation (15-17), whereas reversible mechanisms include principally cell cycle arrest and cellular quiescence (18). The potential anticancer effect reversibility of pimozide would have direct effects on the dosage and administration cycle of the drug. Elucidation of these dynamics can accelerate the clinical translation of pimozide for oncological applications.

The aim of the present study was to investigate the type of proliferative inhibition produced by pimozide on liver cancer cells. We used cell proliferation assays with pan-caspase and 
RIP (receptor-interacting protein) kinase inhibitors to identify whether the effects of pimozide are associated with apoptosis or necrosis. In addition, we analyzed the effects of pimozide on the cell cycle. Importantly, we assessed the reversibility of the anti-proliferative effects of pimozide on liver cancer cells, the potential associations of the efficacy of pimozide with quiescence and ROS production, and the interaction of pimozide with sorafenib which is a tyrosine kinase inhibitor used to treat cancers affecting various organs.

\section{Materials and methods}

Cell lines and culture. The human liver cancer cell lines Sk-Hep1, Huh7 and HepG2 (provided by the Guangdong Provincial Key Laboratory of Liver Disease Research, Guangzhou, China) were cultured in Dulbecco's modified Eagle's medium (DMEM; Gibco; Thermo Fisher Scientific, Inc.) containing $10 \%$ fetal bovine serum (FBS; Sigma-Aldrich; Merck $\mathrm{KGaA})$, penicillin $(100 \mathrm{U} / \mathrm{ml})$ and streptomycin $(100 \mu \mathrm{g} / \mathrm{ml})$. The cell lines were maintained in $5 \% \mathrm{CO}_{2}$ at $37^{\circ} \mathrm{C}$. Huh7 and Sk-Hep1 cell lines were authenticated by short tandem repeat profiling analysis conducted by Procell Life Science \& Technology Co., Ltd. (Wuhan, China).

Cell viability assay. Cell proliferation was assessed using the Cell Counting Kit-8 (CCK-8) assays (MedChem Express, Monmouth Junction, NJ, USA). Liver cancer cells were plated in 96-well plates (10,000 cells/well) and exposed to different concentrations of pimozide ( 5 and $10 \mu \mathrm{M}$ ) (MedChem Express) for $48 \mathrm{~h}$. Kit-provided solution was added to each well and incubated at $37^{\circ} \mathrm{C}$ for $4 \mathrm{~h}$. Absorbance at $450 \mathrm{~nm}$ was detected by a multi-well plate reader (Bio-Rad Laboratories, Inc., Hercules, CA, USA). In addition, Sk-Hep1 and Huh7 cells were treated with $10 \mu \mathrm{M}$ pimozide alone or with combination plus pan-caspase inhibitor Z-VAD-FMK $(10 \mu \mathrm{M})$ or RIP kinase inhibitor necrostain $1(10 \mu \mathrm{M})$ or $2 \mathrm{mM}$ NAC (MedChem Express) for $48 \mathrm{~h}$ before being subjected to cell viability assay. In addition, Sk-Hep1 and Huh7 cells were treated with the $5 \mu \mathrm{M}$ pimozide alone, sorafenib (MedChem Express) alone or combination for $48 \mathrm{~h}$ and then were subjected to cell viability assay.

Colony formation assay. The colony formation assay was performed as described previously (19). Liver cancer cells (500 cells/well) were plated in 10\% FBS medium and treated with different concentrations of pimozide (5 and $10 \mu \mathrm{M})$. After incubation for 2 weeks, the cells were fixed with methanol and stained with crystal violet. Colony morphology was imaged under a stereomicroscope (magnification x100). The colonies, defined as a cluster of at least 50 cells, were counted.

Cell cycle distribution assay. Cell cycle distribution was determined by propidium iodide (PI, Sigma-Aldrich; Merck $\mathrm{KGaA}$ ) staining. Equal cell aliquots were seeded in 6-well plates and treated with pimozide for $48 \mathrm{~h}$. The cells were harvested, washed with phosphate-buffered saline containing $0.1 \%$ bovine serum albumin, and vortexed with cold absolute ethanol. After addition of PI buffer $(40 \mu \mathrm{g} / \mathrm{ml}$, containing $100 \mu \mathrm{g} / \mathrm{ml}$ RNase), cells were analyzed by flow cytometry (CytoFLEX, Beckman Coulter, Inc.).
For quiescent cell detection, cells were washed, fixed with $0.5 \mathrm{ml}$ of $4 \%$ paraformaldehyde in phosphate-buffered saline, and then incubated with an equal volume of $0.2 \%$ Triton-X. The fixed and permeabilized cells were washed, resuspended in staining buffer, and stained with Ki-67-FITC (BD Pharmigen). The stained cells were washed and resuspended in propidium iodide (PI) staining buffer. Cells in G0 phase were analyzed by flow cytometry (CytoFLEX, Beckman Coulter, Inc.) based on $\mathrm{Ki}-67$ negativity and PI incorporation.

Annexin V/PI staining apoptosis assay. Apoptotic cells were detected with an Annexin V-FITC/PI Apoptosis Detection kit (Absin Bioscience, Shanghai, China) according to the manufacturer's instructions. Briefly, cells were treated with $10 \mu \mathrm{M}$ pimozide for $48 \mathrm{~h}$, submitted to Annexin V-FITC/PI staining, and then analyzed by flow cytometry (CytoFLEX, Beckman Coulter, Inc.).

ROS assay and ROS inhibition. ROS products were detected with 2,7-dichlorofluorescein diacetate (DCFH-DA), a fluorescent dye (Beyotime, Jiangsu, China). Briefly, after $10 \mu \mathrm{M}$ pimozide treatment for $48 \mathrm{~h}$, cells were washed and incubated with DCFH-DA $(10 \mu \mathrm{M})$ for $30 \mathrm{~min}$ at $37^{\circ} \mathrm{C}$ in the dark. Then, cells were washed twice with DMEM and analyzed by flow cytometry at an excitation/emission wavelength of 488/525 nm. ROS production was inhibited with $\mathrm{N}$-acetyl-l-cysteine (NAC).

Western blotting. Equal amounts of protein $(20 \mu \mathrm{g} / \mathrm{well})$ collected from cells with RIPA lysis buffer were subjected to sodium dodecyl sulfate-polyacrylamide gel electrophoresis (SDS-PAGE) and transferred to polyvinylidene difluoride membranes (Merck Millipore, Billerica MA, USA). Blots were detected using primary antibodies against cell division protein kinase 6 (CDK6) (cat. no. ab124821; dilution 1:1,000), p-Rb (cat. no. ab173289; dilution 1:1,000) (Abcam, Cambridge, MA, USA), p27 (\#3686; dilution 1:1,000), cyclin D1 (\#2978; dilution 1:1,000), STAT3 (\#12640; dilution 1:1,000), phosphorylated (p)-STAT3 (at tyrosine 705) (\#9145; dilution 1:1,000), ERK1/2 (extracellular signal-regulated kinase 1 and 2) (\#4695; dilution 1:1,000), phosphorylated (p)-ERK1/2 (\#4376; dilution 1:1,000) (Cell Signaling Technology, Beverly, MA, USA) and $\beta$-actin (cat. no. sc-47778; dilution 1:1,000) (Santa Cruz Biotechnology, Santa Cruz, CA, USA). Antibody binding was detected with an enhanced chemiluminescence kit (Immobilon Western Chemilum HRP Substrate, WBKLS0500, Merck Millipore).

Statistical analysis. The data were analyzed in GraphPad Prism 6.0 (GraphPad Software, Inc., San Diego, CA, USA) and are presented as means with standard deviations (SDs). Student's t tests or analysis of one-way variance (ANOVA) followed by Dunnett's post hoc test for multiple comparisons were applied to assess the statistical differences between groups. P-values $<0.05$ were considered significant.

\section{Results}

The anti-proliferative effect of pimozide on liver cancer cells is independent of drug-induced cell death. Proliferative assays showed that pimozide inhibited the growth of Sk-Hep1, Huh7 and HepG2 liver cancer cells in a dose-dependent 

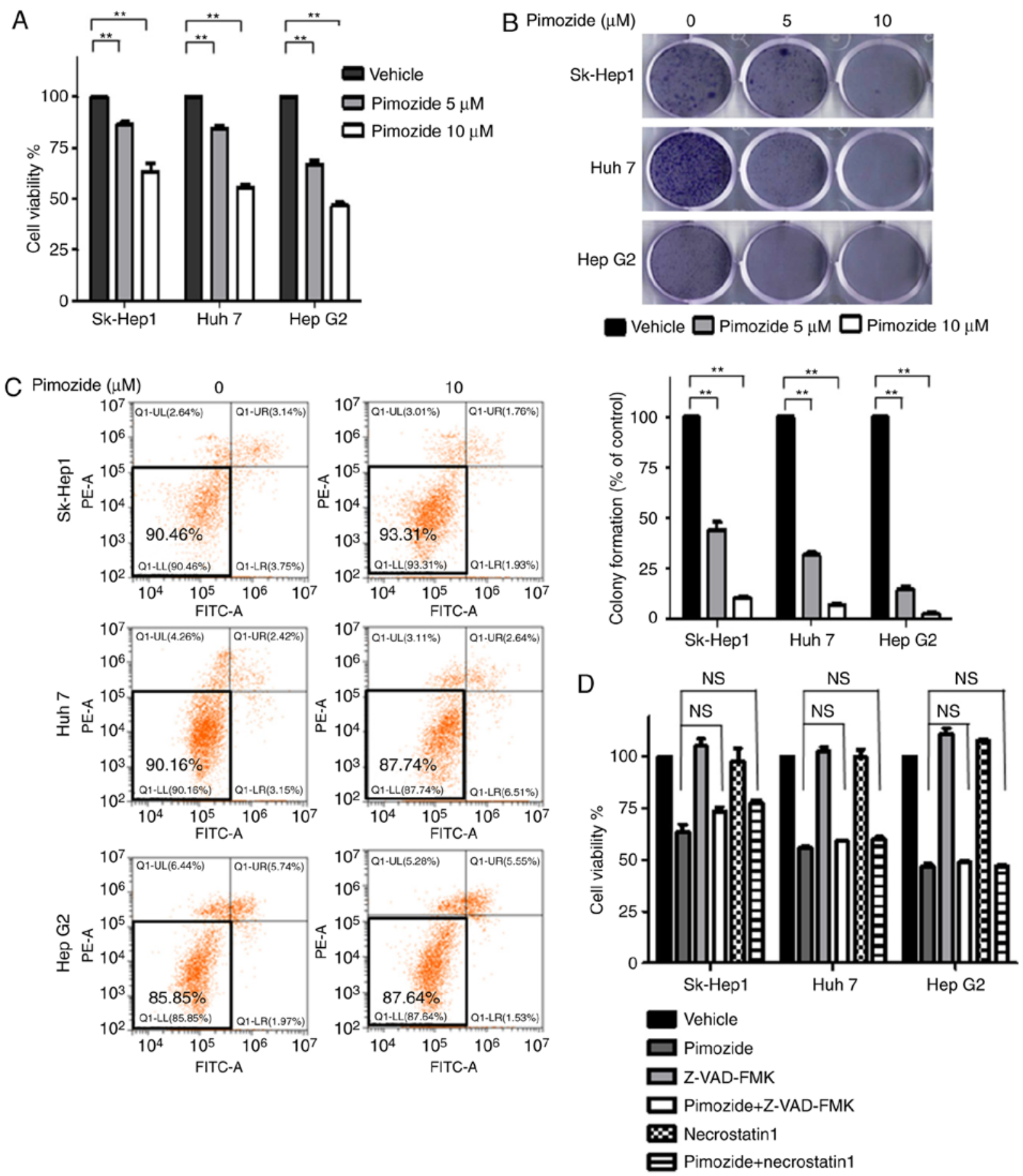

Figure 1. The anti-proliferative effect of pimozide on liver cancer cells is not associated with cell death. Sk-Hep1, Huh7 and HepG2 cells were incubated with 5 and $10 \mu \mathrm{M}$ pimozide for $48 \mathrm{~h}$ for cell viability (A) and colony formation (B) assays. (C) Apoptotic cells detected by Annexin V/PI staining. (D) Treatment of liver cancer cells with $10 \mu \mathrm{M}$ pimozide alone or in combination with the pan-caspase inhibitor Z-VAD-FMK (10 $\mu \mathrm{M}$ ) or the RIP kinase inhibitor necrostain 1 $(10 \mu \mathrm{M})$ for $48 \mathrm{~h}$ before being subjected to cell viability assays. Representative images from one of three independent experiments are shown. Mean values are shown with SDs of three independent experiments; ${ }^{* *} \mathrm{P}<0.01$, compared to the control. NS, not significant.

manner (Fig. 1A). Colony formation assays showed similar inhibition of proliferation across the three cell lines (Fig. 1B). Annexin-V-FITC/PI double staining cell apoptosis assays showed that $10 \mu \mathrm{M}$ pimozide did not induce obvious apoptosis in the liver cancer cells (Fig. 1C). Cell proliferation assays further showed that pan-caspase and RIP kinase inhibitors did not attenuate pimozide inhibition of cell growth in liver cancer cells (Fig. 1D), indicating that the suppression of proliferation was not associated with apoptosis or necrosis.
Pimozide increases cell cycle arrest in HCC cells. As shown in Fig. 2A, PI staining revealed that $14 \mathrm{~h}$ of $10 \mu \mathrm{M}$ pimozide treatment significantly increased the number of cells in the G0/G1 phase in the Sk-Hep1 (68.97 to 77.54\%; $\mathrm{P}<0.01)$ and Huh7 cells (57.47 to $76.84 \%$ ). Western blots showed reduced expression of the cell cycle markers CDK6, $\mathrm{p}-\mathrm{Rb}$ and cyclin D1 together with markedly increased p27 levels (Fig. 2B). These results indicated that the anti-proliferative effect of pimozide is associated with cell cycle arrest. 
A Pimozide $(\mu \mathrm{M})$
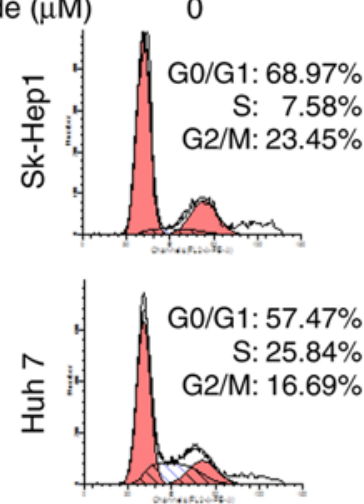

10
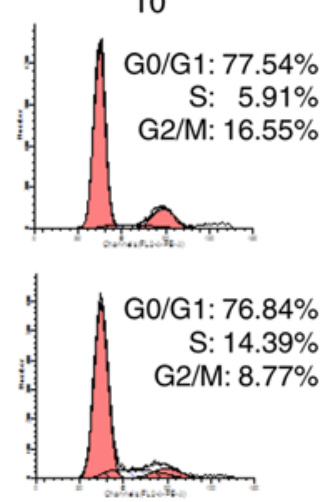

B

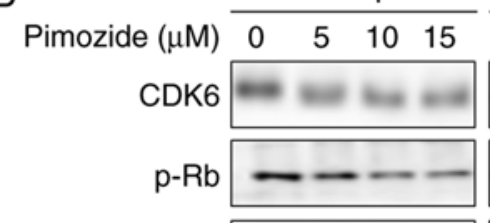

Huh 7
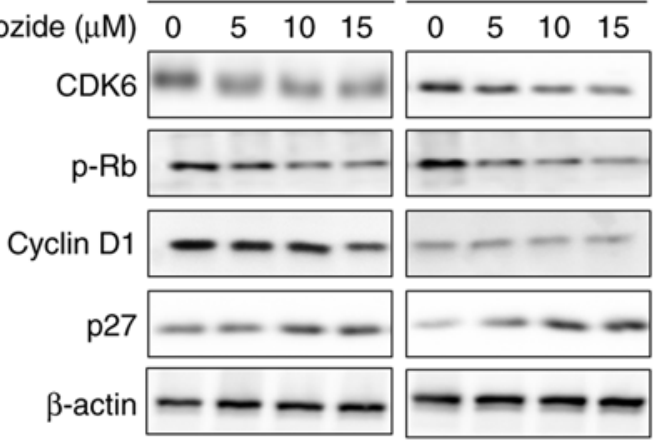

Figure 2. Pimozide induces G0/G1 phase cell cycle arrest in HCC cells. (A) Cell cycle phase distribution of PI-stained Sk-Hep1 and Huh7 cells following treatment with $10 \mu \mathrm{M}$ pimozide was determined by flow cytometry. Representative images from one of three independent experiments are shown. (B) Western blot analysis of cell cycle-related gene expression following $0,5,10$ and $15 \mu \mathrm{M}$ pimozide. Cell extracts were probed with antibodies against CDK6, p-Rb, cyclin D1 and p27 proteins. $\beta$-actin was used as a loading control. HCC, hepatocellular carcinoma; CDK6, cyclin dependent kinase 6.

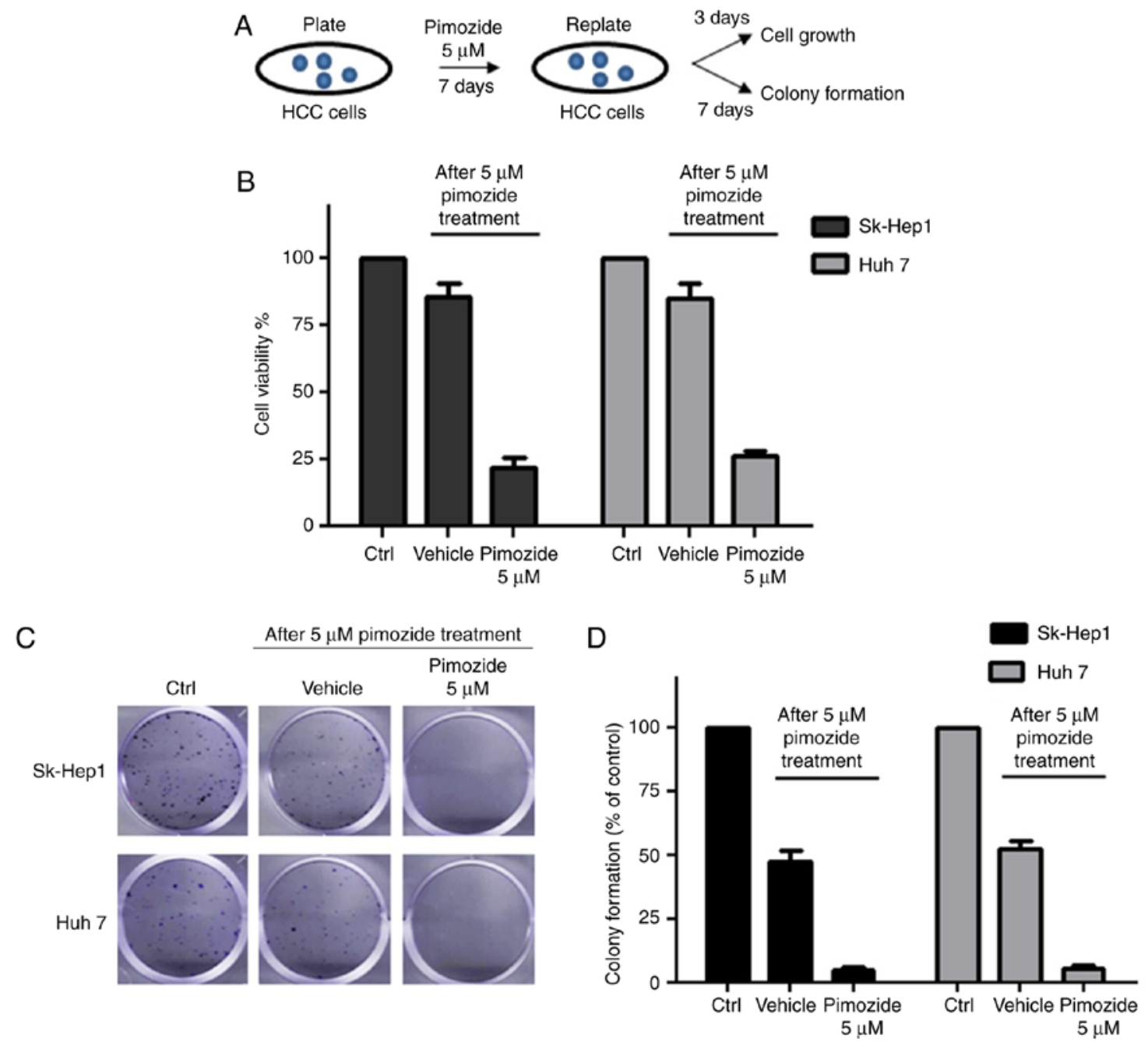

Figure 3. Pimozide induces reversible proliferative suppression in HCC cells. (A) Experimental design diagram: $5 \mu \mathrm{M}$ pimozide, Sk-Hep1 and Huh7 cells were re-plated for cell proliferative and colony formation assays. Analyses of cell proliferative (B) and colony formation (C) assays of Sk-Hep1 and Huh7 cells after pimozide treatment. (D) For the colony formation assay, the numbers of crystal violet-stained colonies were counted. The results were from 3 independent experiments. HCC, hepatocellular carcinoma.

Reversibility of proliferative suppression by pimozide due to quiescence. After treatment with $5 \mu \mathrm{M}$ pimozide, $\mathrm{HCC}$ cells were re-plated in preparation for cell proliferative and colony formation assays, as illustrated in Fig. 3A. Increased cell counts and colony formation were observed in the pimozide-treated Sk-Hep1 and Huh7 cells following pimozide withdrawal, 

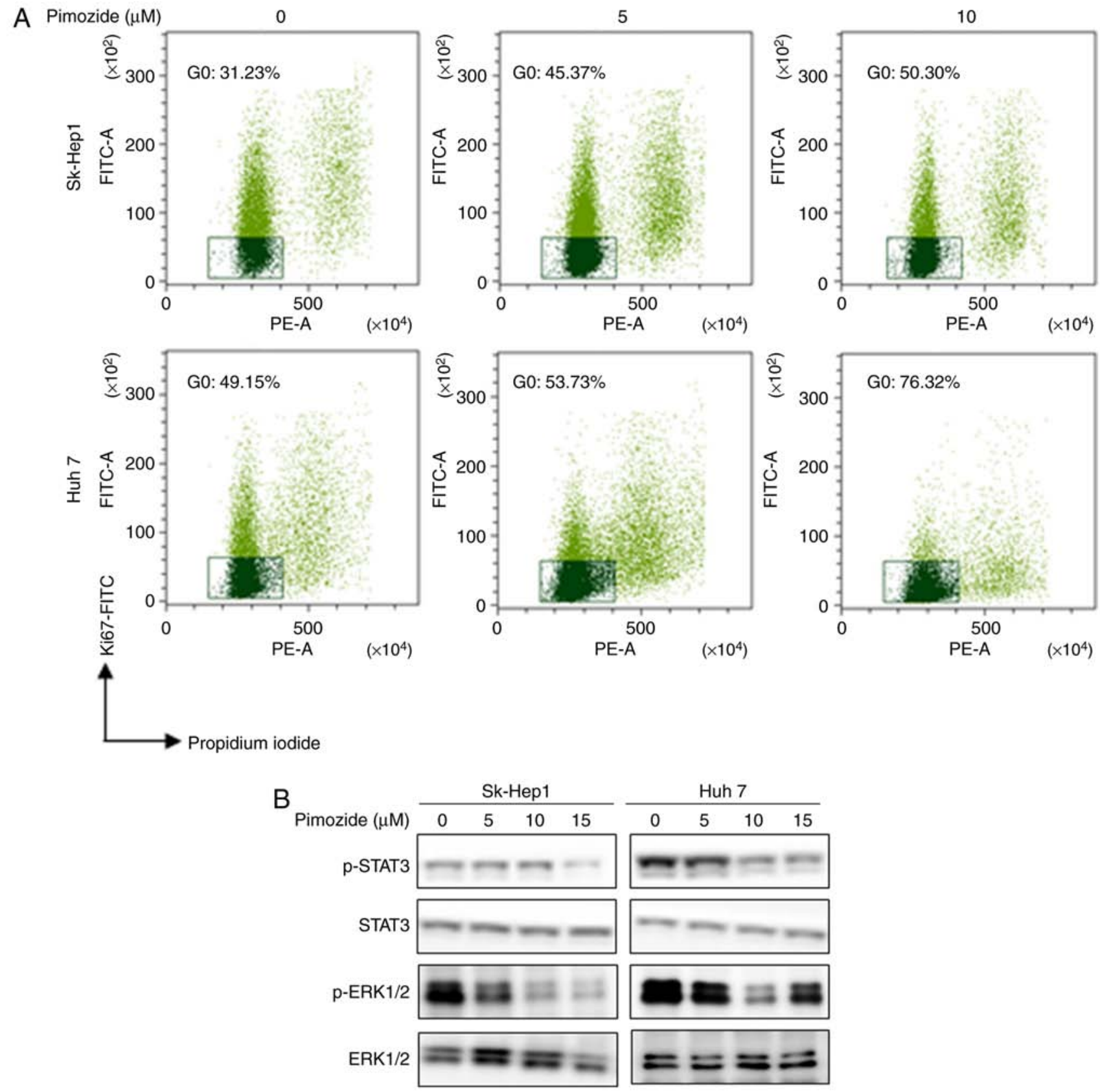

Figure 4. Pimozide induces cellular quiescence in HCC cells. (A) Sk-Hepl and Huh7 cells were incubated with 0,5 and $10 \mu \mathrm{M}$ pimozide for $48 \mathrm{~h}$ and subjected to Ki-67-FITC/PI staining to detect quiescent (G0 phase) cells. Representative flow cytometry data illustrating the Ki67-/PI- population gates for the G0 phase are shown with representative images from one of three independent experiments. (B) Western blot analysis of p-STAT3, STAT3, p-ERK1/2 and ERK1/2 in HCC cells after $0,5,10$ and $15 \mu \mathrm{M}$ pimozide treatment for $48 \mathrm{~h}$. HCC, hepatocellular carcinoma.

demonstrating restoration of proliferation and colony forming abilities (Fig. 3B and C). Following treatment with $5 \mu \mathrm{M}$ pimozide for 1 week, Sk-Hep1 cells showed a decrease of $96.6 \pm 1.1 \%$ in colony number (Fig. 3D), while Sk-Hep1 cells without pimozide treatment displayed significant ability of colony formation $(48.6 \pm 2.3 \%)$. Similar results were achieved in the Huh7 cells.

Ki-67 labelling, PI staining, and flow cytometry showed that pimozide increased the portion of cells in the quiescent G0 phase in a dose-dependent manner (Fig. 4A). After $10 \mu \mathrm{M}$ pimozide treatment, Sk-Hep1 (31.23 to 50.30\%) and Huh7 (49.15 to $76.32 \%$ ) cells had significantly increased percentages of cells in the G0 phase. Western blot analysis showed that pimozide reduced p-STAT3 and p-ERK1/2 levels (Fig. 4B), indicating that pimozide induced HCC cell quiescence.

Pimozide induces ROS generation in HCC cells. Flow cytometry after pimozide exposure (10 $\mu \mathrm{M}$ for $48 \mathrm{~h})$ demonstrated increased ROS levels in the Sk-Hep1 and Huh7 cells and this effect was attenuated by the ROS scavenger NAC (Fig. 5A). NAC also reversed the pimozide-mediated inhibition of Sk-Hep1 and Huh7 cell proliferation (Fig. 5B).

Pimozide enhances the proliferative inhibition induced by tyrosine kinase inhibitor in HCC cells. Cell viability (Fig. 6A) and colony formation assays (Fig. 6B and C) showed that $5 \mu \mathrm{M}$ 

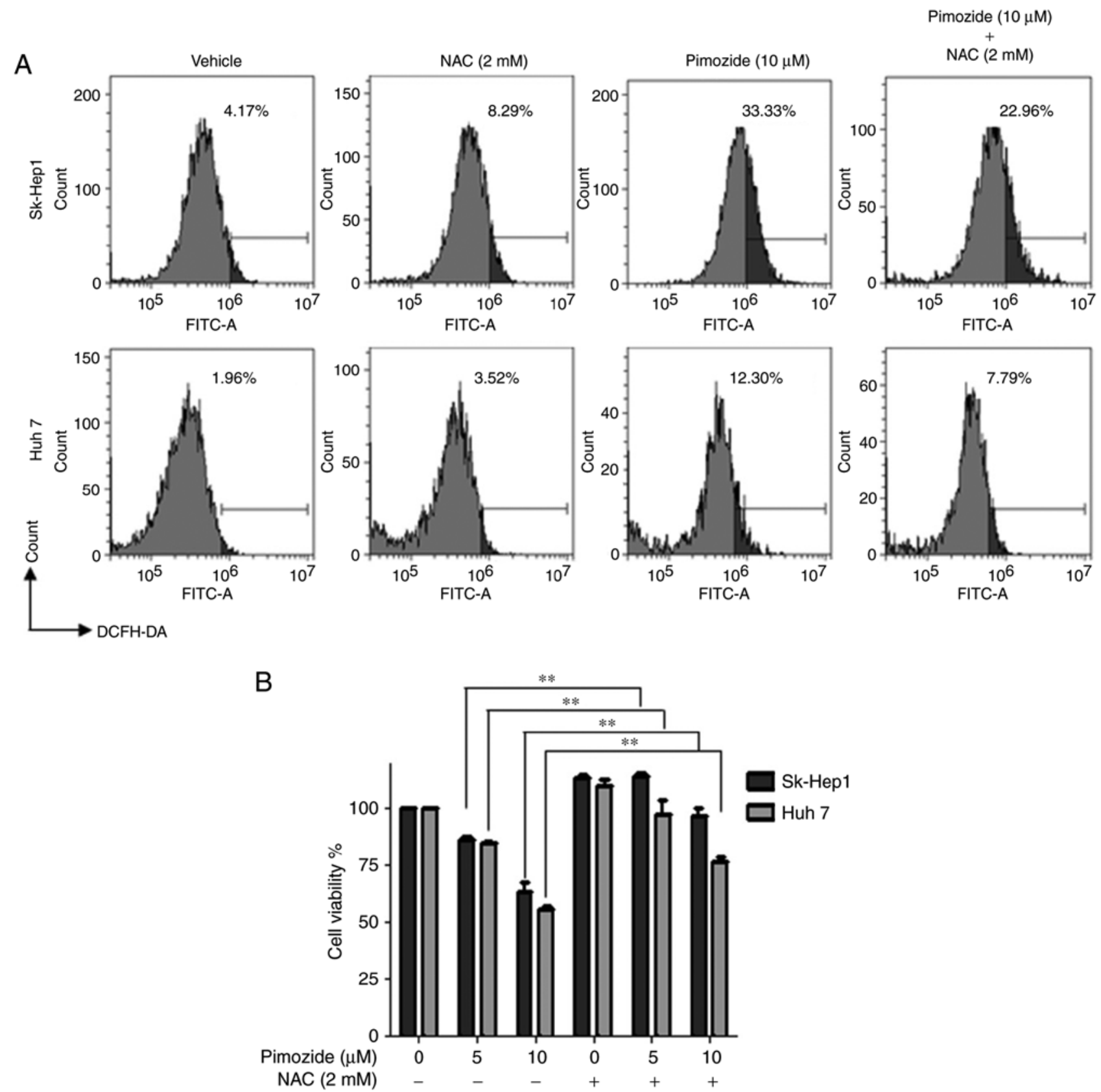

Figure 5. Pimozide treatment increases ROS generation in HCC cells. (A) Sk-Hep1 and Huh7 cells were treated with $10 \mu \mathrm{M}$ pimozide, $2 \mathrm{mM}$ NAC, or both for $48 \mathrm{~h}$, and then stained with DCFH-DA. (A) Analysis of ROS production intensity among assayed cell populations. Representative images from one of three independent experiments are shown. (B) CCK- 8 cell viability assay. Mean values from three independent experiments are shown with SDs, ${ }^{* *} \mathrm{P}<0.01$, compared to the control. ROS, reactive oxygen species; HCC, hepatocellular carcinoma; NAC, N-acetyl-1-cysteine.

pimozide significantly enhanced the anticancer effect of $1 \mu \mathrm{M}$ sorafenib in the Sk-Hep1 and Huh7 cell lines.

\section{Discussion}

Drug effect reversibility is an important factor in determining drug administration mode for optimizing efficacy and persistence while avoiding adverse secondary effects (20). The same drug may produce different effects on different types of tumor cells, mainly due to the particular cell type's genetic background (14). Although pimozide was found to promote the apoptosis of breast cancer $(10,21,22)$, prostate cancer and melanoma cells (23), our data showed that pimozide did not promote apoptosis of liver cancer cells. Moreover, the inhibition of liver cancer cell proliferation by primozide was not affected by pan-caspase or RIP kinase inhibition. These results indicate that the mechanism of the anti-proliferative effects of pimozide on liver cancer cells may not involve drug-induced cell death.

The present observation of the reversible suppression of liver cancer cell proliferation was associated with pimozide-induced cellular quiescence (the resting, non-proliferative G0 phase of the cell cycle) $(24,25)$. Quiescence has been posited to be a drug effect-escape mechanism (26). However, while in this dormant state, cancer cells are not causing harm (27). Because proliferative suppression due to cellular quiescence is transient 
A

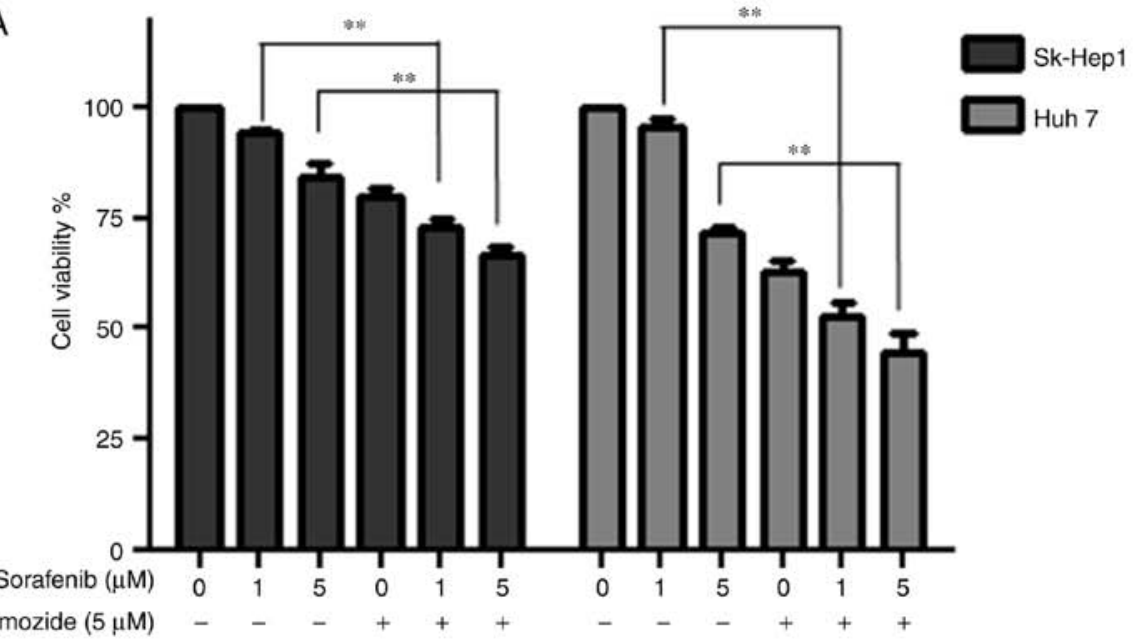

B Sorafenib $(1 \mu \mathrm{M})$
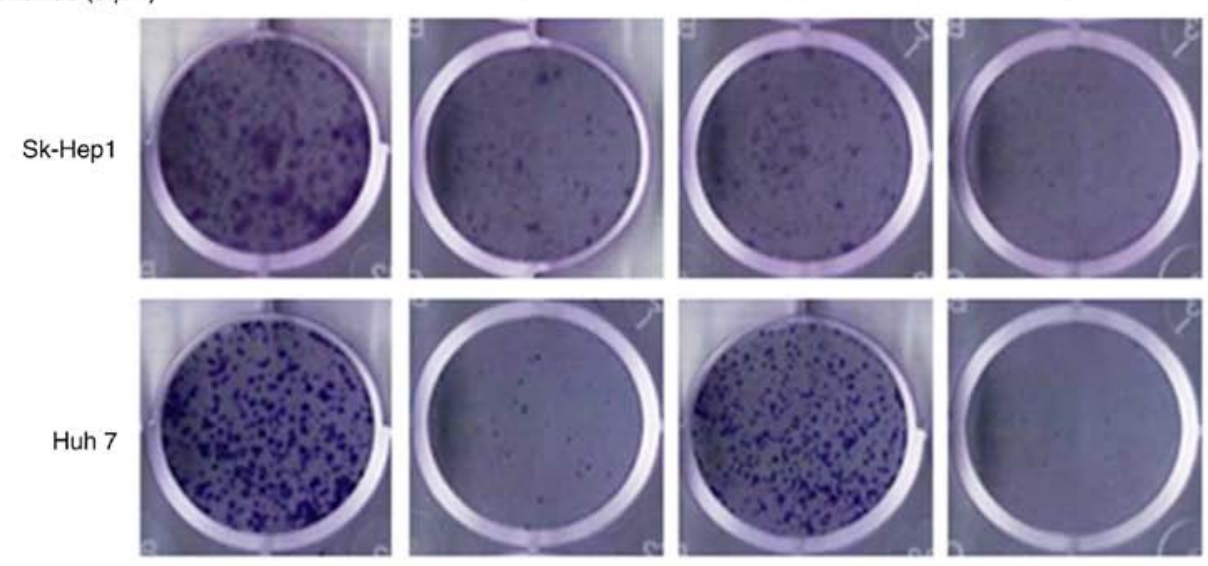

C

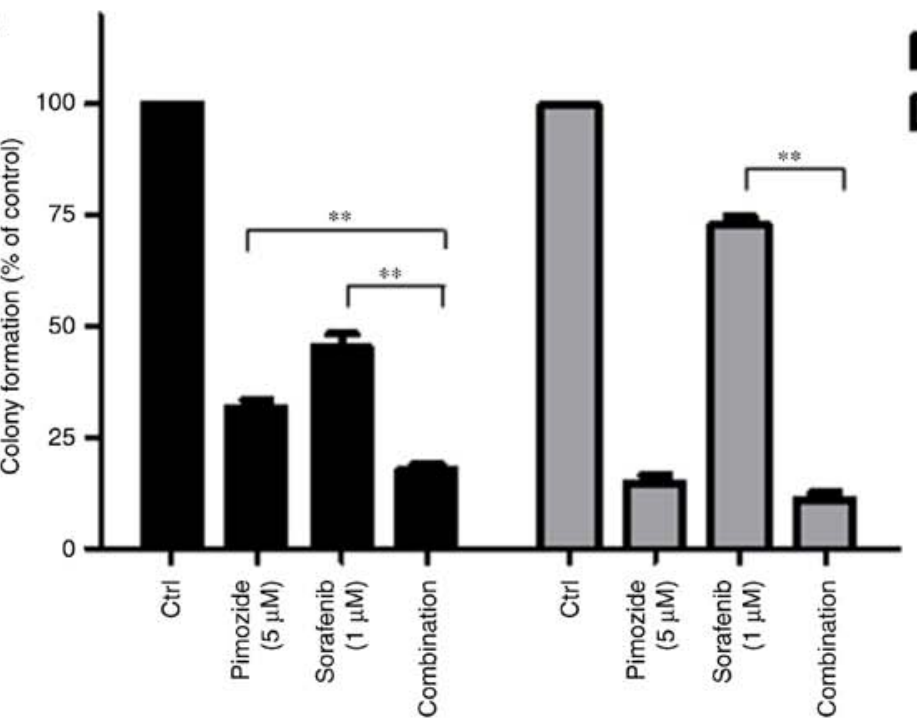

Figure 6. Pimozide enhances the proliferative inhibition of sorafenib in HCC cells. Sk-hep1 and Huh7 cells were treated with $5 \mu \mathrm{M}$ pimozide, sorafenib, or both, and then subjected to cell viability (A) and colony formation (B) assays. Representative images from one of three independent experiments are shown. (C) Mean values from three independent experiments are shown with SDs. The data are summarized from 3 independent experiments, ${ }^{* *} \mathrm{P}<0.01$, compared to the control. HCC, hepatocellular carcinoma.

and reversible, HCC cells will return to a proliferating state if they re-enter the cell cycle (28).

With respect to the mechanism, it was found that HCC cellular quiescence was associated with reduced phosphorylation of STAT3 and ERK1/2. The time-sensitive antitumor activity of pimozide observed suggests that long-term maintenance of pimozide would be needed to maintain its anticancer efficacy. Because pimozide is a well-known antagonist of serotonin 5-hydroxytryptamine receptor 7 (5HT7), we examined 5HT7 expression in liver cancer cell lines and found that 
they indeed had elevated 5HT7 expression (data not shown). Further research is needed to probe the association of 5HT7 expression with cellular quiescence in $\mathrm{HCC}$ cells.

Pimozide is an FDA approved drug shown to have no adverse effects on hepatic or hematopoietic cells. Pimozide is well tolerated in mice without significant effects on body weight $(7,29)$. To date, a lethal dose for pimozide in humans has not been established. The median lethal doses for pimozide in mice and rats are 228 and $5,120 \mathrm{mg} / \mathrm{kg}$, respectively [DrugBank no. DB01100 (30)]. The dose of pimozide used in this study is substantially lower than commonly used clinical doses. Therefore, there is good reason to be optimistic that pimozide may be a safe drug for treating cancer.

Drug repurposing requires a new drug application clinical entry point (31). For example, metformin, a biguanide-class antidiabetic medication, was repurposed as an adjuvant therapy for breast (32) and bladder cancer (33). Similarly, we have been exploring the potential of pimozide to be an adjuvant anticancer therapy. Given the mediocre clinical efficacy of sorafenib alone in patients with HCC, it is hoped that its efficacy may be enhanced by combining it with another intervention (34). Previously, carfilzomib has been shown to work synergistically with sorafenib in inhibiting the proliferation, survival and metastasis of HCC cells in vitro (35). Here, we showed that pimozide can enhance the proliferative inhibition of sorafenib in HCC cells, suggesting that it has the potential to improve HCC outcomes as an adjuvant therapy.

In conclusion, suppression of liver cancer cell proliferation by pimozide was found to involve promotion of cellular quiescence and to be reversible. Furthermore, pimozide was shown to act synergistically with sorafenib, suggesting that it should be further explored as an adjuvant therapy for use with anticancer drugs such as sorafenib in liver cancer treatment with its dosage respecting the reversibility of its effects.

\section{Acknowledgements}

We thank the other members of the laboratory of Ji Kunmei for their critical comments.

\section{Funding}

This work was supported by the National Natural Science Foundation of China (81602595 to JJC), the Natural Science Foundation of Shenzhen City (JCY20170818142053544 to JJC), the Medical Scientific Research Foundation of Guangdong Province (A2019522 to JJC) and 2016 Discipline Construction of Shenzhen City.

\section{Availability of data and materials}

The datasets used during the present study are available from the corresponding author upon reasonable request.

\section{Authors' contributions}

JJC and KJ conceived and designed the study. JJC and LNZ collected and analyzed the data. JJC, LNZ NC and ZZ performed the experiments. JJC and $\mathrm{KJ}$ were responsible for the reagents/materials/analysis tools. JJC and KJ wrote the manuscript. All authors read and approved the manuscript and agree to be accountable for all aspects of the research in ensuring that the accuracy or integrity of any part of the work are appropriately investigated and resolved.

\section{Ethics approval and consent to participate}

Not applicable.

\section{Patient consent for publication}

Not applicable.

\section{Competing interests}

The authors declare that they have no competing interests.

\section{References}

1. Crissien AM and Frenette C: Current management of hepatocellular carcinoma. Gastroenterol Hepatol (N Y) 10: 153-161, 2014.

2. Altekruse SF, McGlynn KA and Reichman ME: Hepatocellular carcinoma incidence, mortality, and survival trends in the united states from 1975 to 2005. J Clin Oncol 27: 1485-1491, 2009.

3. Hoshida Y, Fuchs BC and Tanabe KK: Prevention of hepatocellular carcinoma: Potential targets, experimental models, and clinical challenges. Curr Can Drug Targets 12: 1129-1159, 2012.

4. Cervello M, McCubrey JA, Cusimano A, Lampiasi N, Azzolina A and Montalto G: Targeted therapy for hepatocellular carcinoma: Novel agents on the horizon. Oncotarget 3: 236-260, 2012.

5. Boguski MS, Mandl KD and Sukhatme VP: Drug discovery. Repurposing with a difference. Science 324: 1394-1395, 2009.

6 . Knapp S: New opportunities for kinase drug repurposing and target discovery. Br J Cancer 118: 936-937, 2018.

7. Chen JJ, Cai N, Chen GZ, Jia CC, Qiu DB, Du C, Liu W, Yang Y, Long ZJ and Zhang Q: The neuroleptic drug pimozide inhibits stem-like cell maintenance and tumorigenicity in hepatocellular carcinoma. Oncotarget 8: 17593-17609, 2017.

8. Zhou W, Chen MK, Yu HT, Zhong ZH, Cai N, Chen GZ, Zhang P and Chen JJ: The antipsychotic drug pimozide inhibits cell growth in prostate cancer through suppression of STAT3 activation. Int J Oncol 48: 322-328, 2016.

9. Cai N, Zhou W, Ye LL, Chen J, Liang QN, Chang G and Chen JJ: The STAT3 inhibitor pimozide impedes cell proliferation and induces ROS generation in human osteosarcoma by suppressing catalase expression. Am J Transl Res 9: 3853-3866, 2017.

10. Strobl JS, Melkoumian Z, Peterson VA and Hylton H: The cell death response to gamma-radiation in MCF-7 cells is enhanced by a neuroleptic drug, pimozide. Breast Cancer Res Treat 51: 83-95, 1998.

11. Jia H, Ren W, Feng Y, Wei T, Guo M, Guo J, Zhao J, Song X, Wang M, Zhao T, et al: The enhanced antitumour response of pimozide combined with the IDO inhibitor L-MT in melanoma. Int J Oncol 53: 949-960, 2018.

12. Fako V, Yu Z, Henrich CJ, Ransom T, Budhu AS and Wang XW: Inhibition of wnt/ $\beta$-catenin signaling in hepatocellular carcinoma by an antipsychotic drug pimozide. Int J Biol Sci 12: 768-775, 2016.

13. Ren Y, Tao J, Jiang Z, Guo D and Tang J: Pimozide suppresses colorectal cancer via inhibition of Wnt/ $\beta$-catenin signaling pathway. Life Sci 209: 267-273, 2018.

14. Gonçalves JM, Silva CAB, Rivero ERC and Cordeiro MMR: Inhibition of cancer stem cells promoted by Pimozide. Clin Exp Pharmacol Physiol 46: 116-125, 2019.

15. Yan D, Parker RE, Wang X, Frye SV, Earp HS III, DeRyckere D and Graham DK: MERTK promotes resistance to irreversible EGFR tyrosine kinase inhibitors in non-small cell lung cancers expressing wild-type EGFR family members. Clin Can Res 24: 6523-6535, 2018.

16. Abdallah SM and Hirsh V: Irreversible tyrosine kinase inhibition of epidermal growth factor receptor with afatinib in EGFR activating mutation-positive advanced non-small-cell lung cancer. Curr Oncol 25 (Suppl 1): S9-S17, 2018. 
17. Sagot I and Laporte D: Quiescence, an individual journey. Curr Genet 65: 695-699, 2019.

18. Heldt FS, Barr AR, Cooper S, Bakal C and Novák B: A comprehensive model for the proliferation-quiescence decision in response to endogenous DNA damage in human cells. Proc Natl Acad Sci USA 115: 2532-2537, 2018.

19. Cai N, Xie SJ, Qiu DB, Jia CC, Du C, Liu W, Chen JJ and Zhang Q: Potential effects of $\alpha$-mangostin in the prevention and treatment of hepatocellular carcinoma. J Funct Foods 26: 309-318, 2016.

20. Agarwal SM, Pal D, Gupta M and Saini R: Insight into discovery of next generation reversible TMLR inhibitors targeting EGFR activating and drug resistant T790M mutants. Curr Can Drug Targets 17: 617-636, 2017.

21. Rybczynska M, Spitaler M, Knebel NG, Boeck G, Grunicke H and Hofmann J: Effects of miltefosine on various biochemical parameters in a panel of tumor cell lines with different sensitivities. Biochem Pharmacol 62: 765-772, 2001.

22. Dakir EH, Pickard A, Srivastava K, McCrudden CM, Gross SR, Lloyd S, Zhang SD, Margariti A, Morgan R, Rudl PS and El-Tanani M: The anti-psychotic drug pimozide is a novel chemotherapeutic for breast cancer. Oncotarget 9: 34889-34910, 2018.

23. Strobl JS, Kirkwood KL, Lantz TK, Lewine MA, Peterson VA and Worley JF III: Inhibition of human breast cancer cell proliferation in tissue culture by the neuroleptic agents pimozide and thioridazine. Cancer Res 50: 5399-5405, 1990.

24. Coller HA, Sang L and Roberts JM: A new description of cellular quiescence. PLoS Biol 4: e83, 2006.

25. Aguirre-Ghiso JA: Models, mechanisms and clinical evidence for cancer dormancy. Nat Rev Cancer 7: 834-846, 2007.

26. Alarcón $\mathrm{T}$ and Jensen HJ: Quiescence: A mechanism for escaping the effects of drug on cell populations. J R Soc Interface 8: 99-106, 2011.
27. Fiore APZP, Ribeiro PF and Bruni-Cardoso A: Sleeping beauty and the microenvironment enchantment: Microenvironmental regulation of the proliferation-quiescence decision in normal tissues and in cancer development. Front Cell Dev Biol 6: 59, 2018.

28. Recasens A and Munoz L: Targeting cancer cell dormancy. Trends Pharmacol Sci 40: 30229-30233, 2019.

29. Nelson EA, Walker SR, Xiang M, Weisberg E, Bar-Natan M, Barrett R, Liu S, Kharbanda S, Christie AL, Nicolais M, et al: The STAT5 inhibitor pimozide displays efficacy in models of acute myelogenous leukemia driven by FLT3 mutations. Genes Cancer 3: 503-511, 2012.

30. Wishart DS, Feunang YD, Guo AC, Lo EJ, Marcu A, Grant JR, Sajed T, Johnson D, Li C, Sayeeda Z, et al: DrugBank 5.0: A major update to the DrugBank database for 2018. Nucleic Acids Res 46 (D1): D1074-D1082, 2018.

31. Bhattarai D, Singh S, Jang Y, Hyeon Han S, Lee K and Choi Y: An insight into drug repositioning for the development of novel anti-cancer drugs. Curr Top Med Chem 16: 2156-2168, 2016.

32. Samuel SM, Varghese E, Varghese $S$ and Büsselberg D Challenges and perspectives in the treatment of diabetes associated breast cancer. Cancer Treat Rev 70: 98-111, 2018.

33. El-Arabey AA: Correction to: New insight for metformin against bladder cancer. Genes Environ 40: 16, 2018.

34. Forner A, Reig M and Bruix J: Hepatocellular carcinoma. Lancet 391: 1301-1314, 2018.

35. Jiang C, Xu R, Li XX, Zhou YF, Xu XY, Yang Y, Wang HY and Zheng XFS: Sorafenib and carfilzomib synergistically inhibit the proliferation, survival, and metastasis of hepatocellular carcinoma. Mol Cancer Ther 17: 2610-2621, 2018.

This work is licensed under a Creative Commons Attribution-NonCommercial-NoDerivatives 4.0 International (CC BY-NC-ND 4.0) License. 